\title{
Ventriculostomy Without Decompressive Suboccipital Craniectomy for a Devastating Posterior Fossa Mass Lesion: Doing "Everything" for the Family
}

\author{
David Y. Hwang ${ }^{1,2^{*}}$
}

@ 2020 Springer Science+Business Media, LLC, part of Springer Nature and Neurocritical Care Society

\begin{abstract}
What are neuro-ICU and neurosurgery teams obligated to provide when a family asks for "everything" to be done for a patient with a clearly devastating brain injury? This question comes up routinely in the practice of neurocritical care. Depending on the type and extent of brain injury and the interventions that are theoretically possible, these questions often present a practical strugglewhere prognostic uncertainty, perspectives on acceptable quality of life, respect for the decisional autonomy of an incapacitated patient via substituted judgment by his or her surrogate, and a clinician's level of ethical concern over providing potentially inappropriate treatment often collide.
\end{abstract}

In this issue of Neurocritical Care, Marcellino et al. present a case of a 61-year-old woman initially admitted with severe COVID-19 pneumonia who developed multiorgan failure and then subsequently was found to be comatose off sedation with loss of pupillary reflexes and motor responses on examination [1]. Imaging revealed a large right cerebellar infarct with hemorrhagic conversion, clear evidence of upward herniation, and significant obstructive hydrocephalus. The clinical team held a goals-of-care conversation with the family, who was insistent that all therapeutic measures be undertaken. The neurosurgery team declined a suboccipital decompressive craniectomy given the patient's poor prognosis,

\footnotetext{
*Correspondence: david.hwang@yale.edu

1 Division of Neurocritical Care and Emergency Neurology, Department of Neurology, Yale School of Medicine, PO Box 208018, New Haven, CT 06520, USA

Full list of author information is available at the end of the article
}

but a ventriculostomy was still offered and placed even after the team communicated to the family that a poor outcome for the patient was certain, regardless.

The authors present the subsequent events and sequential neuroimaging of the case as an instructive opportunity to discuss how brain death can develop from a posterior fossa mass lesion: likely compression of the deep venous system from upward herniation, venous congestion, increased intracranial pressure, impedance of cerebral blood flow, total brain infarction. The authors do note that, by the time the patient had progressed to brain death, her ventricles were completely collapsed. This case raises an issue that is often discussed in the neuro-ICU but difficult to study: how much does ventriculostomy worsen upward herniation from an obstructive posterior fossa mass lesion in the absence of surgical decompression? Classic teaching suggests that the risk of exacerbating upward herniation in these situations is significant, although a prior case series (whose authorship overlaps with this case report) does challenge that conventional wisdom [2].

Regardless, for this particular case, the authors themselves state that, "In such a multifaceted brain injury, ventriculostomy will not improve the condition nor provide decompression of the cerebellum" [1]. This raises the related but separate question of whether ventriculostomy itself, as a surgical procedure, should really have been offered at all for this patient in the setting of her initial poor examination, devastating neuroimaging findings, her advanced comorbidities, and the decision to forgo decompression. What are neurointensivists and neurosurgeons obligated to offer when a family is insistent 
that "everything" is done? While multiple studies among a variety of neuro-ICU conditions and the Neurocritical Care Society's own Recommendations for the Critical Care Management of Devastating Brain Injury [3] do warn against early limitations in treatment, it may nevertheless be obvious to clinicians for many severely braininjured patients (including the patient in the case report) that performing an aggressive intervention requested by the family has no chance at improving clinical outcome.

Resolution of clinician-family disagreement over potentially inappropriate treatment is particularly difficult for time-pressured decisions, as it is not practical for such decisions to undergo extensive institutional review (e.g., via a clinical ethics committee, etc.) [4]. An consensus statement on potentially inappropriate treatment from multiple critical care professional societies advises clinicians who find themselves mediating these time-pressured situations to ask themselves the initial question, "Am I certain that this requested treatment is outside the boundaries of accepted practice?" [4]. For the patient in the case report, while the vast majority of neurocritical care specialists would agree that a poor outcome was certain regardless of interventions performed, whether the act of offering a ventriculostomy without decompression was "outside the boundaries of accepted practice" is much more debatable.

Perhaps the equipoise that the neurocritical care community or an individual clinician might feel over this question might also be influenced by the patient's COVID-19 status. After the ventriculostomy was placed in this patient, the patient was treated in the neuro-ICU for approximately another week before progressing to brain death. For a COVID-negative patient destined to the same fate, such additional treatment can raise questions about the utilization of ICU resources; however, the personal risk that the patient presents to bedside nurses, treating physicians, and other patients in the same ICU is minimal. This is not necessarily so for the COVIDpositive patient. Such reality has influenced the balance that patient and surrogate autonomy has played in some clinical decisions-for example, the decision to provide cardiopulmonary resuscitation for a COVID-positive patient with refractory deterioration [5]. In an era where the pandemic has increased the personal risk that health care professionals assume at work and decreased the resources of many intensive care units, perhaps the calculus of offering interventions that are certain not to be of benefit for COVID-positive patients at the request of their family members may be a bit more fraught, even more so than is usual in the neuro-ICU.

\section{Author details \\ ${ }^{1}$ Division of Neurocritical Care and Emergency Neurology, Department of Neurology, Yale School of Medicine, PO Box 208018, New Haven, CT 06520, USA. ${ }^{2}$ Center for Neuroepidemiology and Clinical Neurological Research, Yale School of Medicine, New Haven, CT, USA.}

Conflict of interest

The author declares that he has no conflict of interest.

\section{Publisher's Note}

Springer Nature remains neutral with regard to jurisdictional claims in published maps and institutional affiliations.

Received: 6 November 2020 Accepted: 16 November 2020

Published online: 2 December 2020

References

1. Marcellino C, Braksick SA, Wijdicks EFM. How does the brain die after a massive posterior fossa lesion? Neurocrit Care. 2020. https://doi. org/10.1007/s12028-020-01147-3.

2. Braksick SA, Himes BT, Snyder K, et al. Ventriculostomy and risk of upward herniation in patients with obstructive hydrocephalus from posterior fossa mass lesions. Neurocrit Care. 2018;28(3):338-43.

3. Souter MJ, Blissitt PA, Blosser S, et al. Recommendations for the critical care management of devastating brain injury: prognostication, psychosocial, and ethical management : a position statement for healthcare professionals from the neurocritical care society. Neurocrit Care. 2015;23(1):413.

4. Bosslet GT, Pope TM, Rubenfeld GD, et al. An official ATS/AACN/ACCP/ ESICM/SCCM policy statement: responding to requests for potentially inappropriate treatments in intensive care Units. Am J Respir Crit Care Med. 2015;191(11):1318-30

5. Kramer DB, Lo B, Dickert NW. CPR in the covid-19 era-an ethical framework. N Engl J Med. 2020;383(2):e6. 